ARTIGO ORIGINAL ORIGINAL ARTICLE

Palavras-chave:

avaliação da tecnologia biomédica, trombose venosa, neoplasias

\title{
Análise de custo-efetividade da rivaroxabana versus enoxaparina no tratamento da trombose venosa profunda em pacientes com câncer ginecológico
}

\author{
Cost-effectiveness analysis of rivaroxaban versus \\ enoxaparin in the treatment of deep venous \\ thrombosis in patients with gynecologic cancer \\ Rafael Roberto Leira', Rodrigo Saar da Costa² \\ DOI: 10.21115/JBES.v10.n1.p2-8
}

\begin{abstract}
RESUMO
Objetivo: A trombose venosa profunda (TVP) é uma das principais complicações em pacientes com câncer e tem impacto socioeconômico relevante. Novos anticoagulantes orais inibidores diretos do fator X estão sendo introduzidos na prática clínica, prometendo trazer maior facilidade no seu manejo. Porém, os protocolos clínicos ainda não discutem o emprego deles em pacientes com câncer, principalmente pela falta de evidências. Este artigo avalia o custo-efetividade da rivaroxabana (RVXB) versus enoxaparina (ENOXA) no tratamento da TVP em pacientes com câncer ginecológico. Métodos: Foi realizada uma análise de custo-efetividade sob a perspectiva de um hospital federal do SUS. A partir de um modelo de árvore de decisão, foram considerados os desfechos de efetividade e segurança da RVXB e da ENOXA em tratamento ambulatorial, sendo os dados clínicos extraídos de uma coorte retrospectiva e os custos diretos obtidos das plataformas SIGTAP-SUS e do Ministério da Saúde. Resultados: Sessenta e cinco pacientes foram selecionados e tiveram tempo médio de sete meses de tratamento. O custo de tratamento com a RVXB e a ENOXA foi de R\$1.172,02 e $\mathrm{R} \$ 7.490,49$, respectivamente, com custo incremental de $\mathrm{R} \$ 7.789,61$ por paciente tratado com ENOXA. A robustez do modelo foi testada por análises de sensibilidade determinísticas e a economia de recursos promovida pela rivaroxabana foi mantida em todas as variações. Conclusão: A RVXB neste contexto é a tecnologia dominante no tratamento ambulatorial de pacientes com câncer ginecológico como importante alternativa farmacoterapêutica.
\end{abstract}

\section{Keywords:}

biomedical technology assessment, venous thrombosis, neoplasms

\begin{abstract}
Objective: Deep vein thrombosis (DVT) is one of the main complications in cancer patients with relevant socioeconomic impact. New direct factor $\mathrm{X}$ inhibitor oral anticoagulants are being introduced into clinical practice, promising to bring greater ease in their management. However, the clinical protocols do not yet discuss the use of these in cancer patients mainly due to lack of evidence. This article aims to evaluate the cost-effectiveness of rivaroxaban (RVXB) versus enoxaparin (ENOXA) in the treatment of DVT in patients with gynecological cancer. Methods: A cost-effectiveness analysis was performed from the perspective of a federal hospital SUS. From a decision tree model, the effectiveness and safety outcomes of RVXB and ENOXA in outpatient treatment were considered, with clinical data extracted from a retrospective cohort, and the direct costs obtained from the SIGTAP-SUS and Ministry of Health. Results: 65 patients were selected and had an average time of seven months of treatment. The cost of treatment with RVXB and ENOXA was R\$ 1.172,02 and R\$7.490,49,
\end{abstract}

\footnotetext{
Recebido em: 14/03/2017. Aprovado para publicação em: 19/03/2018

1. Instituto Nacional de Câncer José Alencar Gomes da Silva (Inca).

2. Instituto Nacional de Câncer José Alencar Gomes da Silva (Inca); Instituto COPPEAD - Universidade Federal do Rio de Janeiro (UFRJ).

Declaração de conflito de interesse: Nenhum dos autores recebe qualquer patrocínio da indústria ou participa de qualquer entidade de especialidade ou de pacientes que possa representar conflito de interesse.

Autor correspondente: Rodrigo Saar da Costa. Instituto Nacional de Câncer José Alencar Gomes da Silva. Rua Marquês de Pombal, 125, 70 andar, Centro, Rio de Janeiro, RJ, Brasil. CEP: 20230-240. Telefone: +55 (21) 99654-8981.
} 
respectively, with an incremental cost of $\mathrm{R} \$ 7.789,61$ per patient treated with ENOXA. Robustness of the model was tested by deterministic sensitivity analysis in which the resource saving promoted by rivaroxaban was maintained in all variations Conclusion: The RVXB in this context is the dominant technology in the outpatient treatment of patients with gynecological cancer.

\section{Introdução}

A trombose venosa profunda (TVP) é uma complicação comum em pacientes com câncer, associada a importantes taxas de morbimortalidade e com elevados custos em saúde (Lee et al., 2013). Pacientes com câncer possuem risco quatro vezes maior de desenvolver TVP em comparação com a população em geral (Carrier et al., 2009). Além disso, possuem maior chance de óbito quando comparados a pacientes que têm câncer e não tiveram episódios de trombose ou que só tiveram episódios de trombose e não tiveram câncer (Meis et al., 2007).

Segundo modelo preditivo de Khorana, os cânceres ginecológicos possuem alto risco para o desenvolvimento de trombose. Ele está relacionado à malignidade do próprio câncer, à idade avançada e à necessidade de realização de cirurgia abdominal e pélvica extensa (Khorana et al., 2008).

O tratamento da TVP em pacientes com câncer é limitado em alguns casos, pois as recomendações para o manejo são extrapoladas de ensaios clínicos que em geral não possuem como critérios de inclusão pacientes com câncer (Lee et al., 2013). Os novos anticoagulantes orais são uma possível opção para o tratamento, que tradicionalmente é realizado com antagonistas da vitamina $\mathrm{K}$ e heparinas de baixo peso molecular (HBPM) (Short et al., 2014).

Os novos anticoagulantes orais, como os inibidores diretos do fator $X$, estão sendo introduzidos na prática clínica e trazendo comodidade ao paciente e facilidade no seu manejo quando comparados aos anticoagulantes tradicionais. Alguns estudos têm demonstrado a rivaroxabana (RVXB) como alternativa de escolha, porém, em contextos internacionais e/ou aplicado em pacientes com outras condições clínicas (Diamantopoulos et al., 2010; Silva et al., 2011; Duran et al., 2013).

Em um estudo pivotal, EINSTEIN (2010) demonstrou a não inferioridade da RVXB em termos de eficácia e segurança no tratamento da TVP comparada aos demais anticoagulantes, tendo com subgrupo pacientes com câncer ativo (EINSTEIN Investigators et al., 2010). Porém, o American College of Chest Physicians (CHEST), em recente atualização do seu protocolo clínico para tratamento de tromboembolismo venoso, recomenda o uso de HBPM em pacientes com câncer (Kearon et al., 2016). E a American Society of Clinical Oncology (ASCO) não recomenda o uso dos novos anticoagulantes orais em pacientes com câncer, devido à falta de evidências clínicas (Lyman et al., 2015). No Brasil, as recomendações de protocolos clínicos e diretrizes terapêuticas ainda não discutem o emprego desses novos anticoagulantes em pacientes com câncer, pela falta de evidências clínicas relacionadas a sua eficácia, efetividade e segurança, além da carência de estudos farmacoeconômicos.

A RVXB faz parte da classe dos novos anticoagulantes orais disponíveis para tratamento da TVP. Trata-se de um medicamento ainda muito estudado, e sua indicação pode levar a desfechos e recomendações promissoras para pacientes oncológicos com TVP. Considerando o exposto, faz-se necessário avaliar sua razão de custo-efetividade comparado ao tratamento convencional em pacientes com câncer ginecológico no tratamento da TVP.

\section{Métodos}

Trata-se de um estudo de custo-efetividade, de caráter retrospectivo, realizado em um hospital federal do Sistema Único de Saúde (SUS), com perfil de atendimento em câncer ginecológico. A análise foi conduzida tendo como população-alvo pacientes portadoras de câncer ginecológico, sendo os de maior frequência os cânceres de colo de útero, de ovário e corpo de útero.

Foram incluídas no estudo pacientes portadoras de câncer ginecológico e TVP concomitantemente, com idade acima de 18 anos e que usaram HBPM ou RVXB para o tratamento de TVP por pelo menos três meses consecutivos. Foram excluídas do estudo todas as pacientes que não atenderem aos critérios estabelecidos acima, pacientes cirúrgicas, assim como pacientes que não permaneceram ou foram encaminhadas para tratamento em outros serviços da rede do SUS. A perspectiva adotada para o estudo foi para um órgão público prestador de serviços de saúde.

A RVXB, introduzida na instituição 2014, foi utilizada em pacientes com câncer e diagnóstico de TVP como alternativa à ENOXA, de acordo com protocolos clínicos adaptados de recomendações internacionais (Kearon et al., 2016; Lyman et al., 2015; Streiff et al., 2015). O esquema de tratamento para a RVXB foi de $15 \mathrm{mg}$ a cada 12 horas durante três semanas, seguido de uma dose de $20 \mathrm{mg}$ uma vez ao dia (Bayer, 2015), e para a ENOXA, de $1,5 \mathrm{mg} / \mathrm{kg} / \mathrm{dia}$ ou $1 \mathrm{mg} / \mathrm{kg}$ a cada 12 horas (Sanofi-Aventis, 2015). De acordo com a conduta da instituição, todos os pacientes com TVP aguda são tratados inicialmente com ENOXA seguida de substituição pela RVXB ou manutenção com ENOXA durante um tempo médio de sete meses.

Dessa forma, o horizonte temporal desta análise foi de sete meses, considerando o tempo de tratamento e o surgi- 
mento dos desfechos avaliados, compatível com a proposta do modelo analítico e estudos clínicos pivotais. Não foi necessária aplicação de taxa de desconto em virtude do estreito horizonte temporal.

Foram selecionadas como desfechos primários para esta análise a recorrência de TVP. que está diretamente relacionada com a efetividade, e a ocorrência de sangramento clinicamente relevante, relacionado à segurança, baseado em estudos clínicos pivotais. Após o início de tratamento com o anticoagulante, os desfechos foram extraídos dos registros em prontuário médico. Para o cálculo da efetividade (E) das tecnologias, foi considerado o número de pacientes com probabilidade de ocorrência de TVP recorrente (P). Onde: E = (1 - P) (EINSTEIN Investigators et al., 2010; Carrier et al., 2014; Prandoni et al., 2002). Os critérios de classificação da International Society on Thrombosis and Haemostasis (ISTH) foram utilizados para o desfecho sangramento (Kaatz et al., 2015), e para o desfecho de TVP recorrente foi considerado o critério do algoritmo do CHEST (Bates et al., 2012). O uso de desfechos finalísticos como qualidade de vida ou óbito poderiam causar confundimento, uma vez que estivessem associados ao câncer, e não à TVP.

Os dados clínicos foram obtidos de uma coorte retrospectiva de pacientes em tratamento ambulatorial no hospital, no período de janeiro de 2014 a junho de 2016. Foram levantados dados epidemiológicos dos pacientes com relação a idade, tipo de tumor, tempo de uso dos anticoagulantes, estadiamento durante o primeiro episódio de TVP, realização de cirurgia, de quimioterapia e de radioterapia, sangramento, TVP recorrente e óbito. Todos os desfechos clínicos foram obtidos por meio do prontuário.

Os custos considerados para este estudo correspondem a valores de reembolso aplicados pelo SUS. Foram considerados os custos de tratamento e de desfechos de sangramento e TVP recorrente em nível ambulatorial das tecnologias analisadas. Para esta análise foram considerados somente custos médicos diretos, sendo extraídos do Sistema de Gerenciamento da Tabela de Procedimentos, Medicamentos, Órteses, Próteses e Materiais e Medicamentos Especiais (SIGTAP) e das bases de dados de compras oficiais do Ministério da Saúde disponíveis no sítio www.comprasgovernamentais.gov.br, no período de abril a maio de 2015.

A partir da coleta de dados dos desfechos, foi construída uma árvore de decisão, sendo inseridas as probabilidades relativas a cada evento encontrado, e estes associados aos seus custos. Os pressupostos estruturais adotados para o emprego do modelo assumiram a não recorrência dos desfechos no período de sete meses, sendo sangramento seguido de TVP recorrente a ordem de aparição no modelo (Figura 1).

Foi realizada análise de sensibilidade univariada e bivariada de forma determinística, a partir da variação dos custos dos medicamentos e do grau de incertezas da gravidade dos

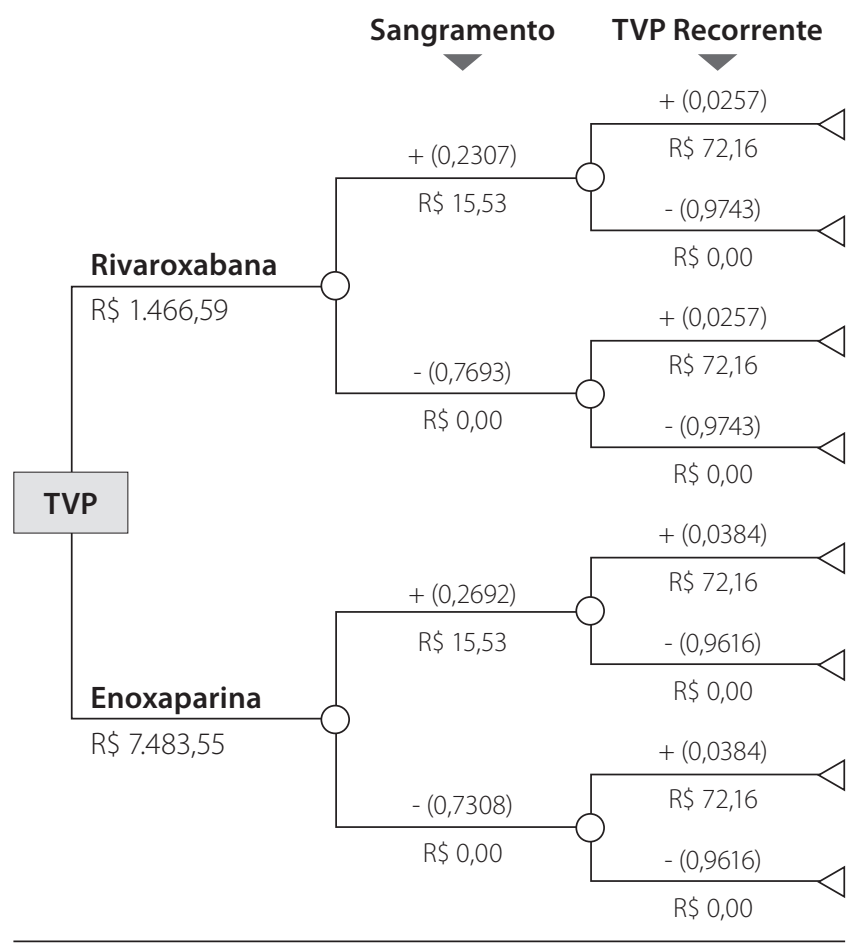

Figura 1. Representação esquemática da árvore de decisão de custo-efetividade da rivaroxabana versus enoxaparina no tratamento da TVP em pacientes com câncer ginecológico.

desfechos (Secoli et al., 2010). Foram propostos três cenários, que tinham como objetivo avaliar variações dos custos dos medicamentos e/ou agravamento dos desfechos primários avaliados. No cenário 1, foi aplicada variação de $25 \%$, de acordo com as recomendações atuais da Ministério da Saúde (Brasil, 2014), no custo de tratamento da tecnologia dominante. Para o cenário 2, o objetivo foi simular o agravamento do desfecho de sangramento maior para a tecnologia dominante, obtido por meio dos ensaios clínicos pivotais (EINSTEIN Investigators et al., 2010; Meyer et al., 2002). Por fim, o cenário 3 considerou o uso de complexo protrombínico para tratamento de sangramento maior e aumento em $50 \%$ do custo da tecnologia dominante. Não foi aplicada a simulação de Monte Carlo e Tornado para esse modelo.

O software utilizado para a construção do modelo e a análise estatística foi o Microsoft Excel ${ }^{\circledR}$ 2010. A unidade monetária utilizada no modelo foi o real (R\$). Este estudo foi aprovado pelo Comitê de Ética em Pesquisa (CEP), CAAE: 54355416.1.0000.5274.

\section{Resultados}

Foram selecionados 65 pacientes, sendo 26 do braço ENOXA e 39 do braço RVXB. A média de idade foi de $57 \pm 13,0$ e 56 $\pm 11,60$ anos para ENOXA e RVXB, respectivamente. O câncer de colo de útero foi o mais prevalente, com $49,20 \%$ dos casos, em ambos os braços, seguido do câncer de ovário, com 
35,30\%. Foi observada prevalência de estadiamentos III e IV em ambos os grupos, caracterizando doença avançada no mesmo período em que tiveram o primeiro episódio de TVP. O estádio IV foi o mais frequente no momento do primeiro episódio de TVP (38,50\%), e este independente do tipo de tumor e do braço de tratamento (Tabela 1).

O tempo de uso do anticoagulante em ambos os braços foi em média de 7,56 \pm 4,69 meses. O primeiro episódio de TVP ocorreu em 30,80\% dos pacientes no momento em que estavam em uso de quimioterapia. Os desfechos sangramento e TVP recorrente apresentaram frequência semelhante entre os braços. Dessa forma, os eventos relacionados à segurança e à efetividade não tiveram diferença estatística significativa ( $p=0,72$ e $p=0,74$, respectivamente). Apenas um paciente de cada braço apresentou ambos os desfechos durante todo o tratamento (Tabela 2).

Os custos considerados para os cálculos de tratamento estão discriminados na Tabela 2, em que foram considerados custos com exames e consultas somados aos custos dos respectivos medicamentos por um período de tratamento de sete meses, e aplicados ao modelo de análise. O custo to-

Tabela 1. Características epidemiológicas dos pacientes com TVP

\begin{tabular}{|c|c|c|}
\hline & Enoxaparina & Rivaroxabana \\
\hline $\mathrm{n}^{\circ}$ de pacientes & 26 & 39 \\
\hline Média de idade em anos (DP) & $57( \pm 13,0)$ & $56( \pm 11,6)$ \\
\hline \multirow[t]{2}{*}{$\begin{array}{l}\text { Média de tempo de uso } \\
\text { do anticoagulante em } \\
\text { meses (mediana) }\end{array}$} & $5,4(5)$ & $8,4(6)$ \\
\hline & \multicolumn{2}{|c|}{ Câncer n (\%) } \\
\hline Câncer de vulva & 7,69 & 2,56 \\
\hline Câncer de colo de útero & 53,80 & 46,15 \\
\hline Câncer de corpo de útero & 11,53 & 10,25 \\
\hline \multirow[t]{2}{*}{ Câncer de ovário } & 26,92 & 41,02 \\
\hline & \multicolumn{2}{|c|}{ Tratamento prévio n (\%) } \\
\hline Cirurgia & 38,46 & 74,35 \\
\hline Quimioterapia & 76,92 & 87,17 \\
\hline Radioterapia & 42,30 & 41,02 \\
\hline \multirow[t]{2}{*}{$\begin{array}{l}\text { Quimioterapia durante } \\
\text { o episódio de TVP }\end{array}$} & 26,92 & 33,30 \\
\hline & \multicolumn{2}{|c|}{$\begin{array}{c}\text { Estadiamento durante o } \\
\text { episódio de TVP (\%) }\end{array}$} \\
\hline । & 11,53 & 10,25 \\
\hline$\|$ & 11,53 & 17,94 \\
\hline III & 42,30 & 28,20 \\
\hline IV & 34,60 & 41,02 \\
\hline$x$ & 0,00 & 2,56 \\
\hline Óbito & 53,80 & 38,46 \\
\hline
\end{tabular}

DP: desvio-padrão. Fonte: elaboração própria. tal foi de $\mathrm{R} \$ 7.483,55$ e de $\mathrm{R} \$ 1.466,59$ para o braço ENOXA e RVXB, respectivamente (Tabela 2).

Os desfechos ocorreram principalmente após dois meses de início do tratamento. No braço ENOXA, 57,10\% dos pacientes apresentaram sangramento e 33,30\%, TVP recorrente, dois meses após o início da terapia. Já no braço RVXB, 33,30\% tiveram sangramento e 100\%, TVP recorrente, após esse período.

Entre os casos de sangramento, 75\% foram observados em pacientes com câncer de colo de útero e em mais de 80\% com estádio avançado da doença. Já para o desfecho TVP recorrente, as pacientes com câncer de ovário foram as que apresentaram maior frequência (75\%), e todos os eventos ocorrem em estadiamentos III e IV.

A árvore de decisão apresentou custo incremental de R\$ 6.318,47 com o uso da ENOXA. Conforme apresentado na Tabela 3, foi verificada uma pequena diferença de efetividade entre os medicamentos ENOXA e RVXB de 0,9616 e 0,9743, respectivamente. O cálculo da razão de custo-efetividade incremental (RCEI) foi de $\mathrm{R} \$ 6.586,67$ para o braço ENOXA, e nesse contexto a RVXB foi dominante.

Tabela 2. Parâmetros aplicados ao modelo da árvore de decisão

\begin{tabular}{|c|c|c|c|}
\hline & $\begin{array}{c}\text { Enoxaparina } \\
n=26(\%)\end{array}$ & $\begin{array}{c}\text { Rivaroxabana } \\
\mathrm{n}=39(\%)\end{array}$ & p-valor \\
\hline Sangramento & 26,90 & 23,00 & 0,72 \\
\hline TVP recorrente & 3,84 & 2,56 & 0,74 \\
\hline Efetividade & $\begin{array}{c}(1-0,0384) \\
0,9616\end{array}$ & $\begin{array}{c}(1-0,0256) \\
0,9743\end{array}$ & - \\
\hline Custos unitários (R\$) & & & Referência \\
\hline $\begin{array}{l}\text { Preço médio dos } \\
\text { medicamentos }\end{array}$ & 17,52 & $11,42 / 5,71^{*}$ & BDOMS \\
\hline Dopplerfluxometria & 43,50 & 43,50 & SIGTAP \\
\hline $\begin{array}{l}\text { Consulta médica } \\
\text { - atendimento } \\
\text { de urgência }\end{array}$ & 11,00 & 11,00 & SIGTAP \\
\hline $\begin{array}{l}\text { Tratamento de } \\
\text { sangramento }\end{array}$ & 15,53 & 15,53 & SIGTAP \\
\hline $\begin{array}{l}\text { Tratamento de } \\
\text { TVP recorrente }\end{array}$ & 72,16 & 72,16 & $\begin{array}{l}\text { SIGTAP/ } \\
\text { BDOMS }\end{array}$ \\
\hline Exame e consultas ( $n$ ) & & & Referência \\
\hline $\begin{array}{l}\text { Média de exame } \\
\text { Dopplerfluxometria }\end{array}$ & 2 & 2 & BDOMS \\
\hline $\begin{array}{l}\text { Média de consultas } \\
\text { médicas }\end{array}$ & 4 & 6 & BDOMS \\
\hline $\begin{array}{l}\text { Custo do tratamento } \\
\text { total (por } 7 \text { meses) }\end{array}$ & $7.483,55$ & $1.466,59$ & - \\
\hline
\end{tabular}

BDOMS - Base de Dados Oficial do Ministério da Saúde (www.comprasgovernamentais.gov.br), acessada no primeiro semestre de 2015. * Custo do tratamento nos primeiros 21 dias foi de $R \$ 11,42$, seguido de $R \$ 5,71$ a partir do $21^{\circ}$ dia. DP: desvio-padrão. Fonte: Elaboração própria. 
Tabela 3. Razão de custo-efetividade incremental entre RVXB e ENOXA

\begin{tabular}{lcc}
\hline & $\begin{array}{c}\text { Enoxaparina } \\
\text { (R\$) }\end{array}$ & $\begin{array}{c}\text { Rivaroxabana } \\
\text { (R\$) }\end{array}$ \\
\hline Custo & $7.490,49$ & $1.172,02$ \\
\hline Custo incremental & $6.318,47$ & - \\
\hline Efetividade & 0,9616 & 0,9743 \\
\hline Efetividade incremental & $-0,0127$ & - \\
\hline Custo-efetividade & $7.789,61$ & $1.202,94$ \\
\hline RCEI & - & DOMINANTE \\
\hline
\end{tabular}

Fonte: Elaboração própria.

A análise de sensibilidade foi aplicada com o objetivo de propor cenários desfavoráveis à tecnologia dominante e testar a robustez do modelo. Foram aplicadas variação de aumento de custos da tecnologia avaliada em $25 \%$ e $50 \%$ e de simulações de agravamento do desfecho de sangramento, contudo em todos os cenários a RVXB permaneceu dominante (Tabela 4).

\section{Discussão}

A incidência de TVP em pacientes com câncer, de acordo com alguns estudos internacionais é de 20\% (Heit et al., 2002; Brose \& Lee et al., 2008). Em um estudo realizado no Instituto Nacional de Câncer (Inca), observou-se que o evento de TVP ocorreu em 7,3\% das pacientes portadoras de câncer ginecológico em estádio IIIB, com redução da sobrevida quando comparadas com as pacientes sem evento tromboembólico (Renni et al., 2011).

Foram avaliados 65 prontuários de pacientes com câncer ginecológico e que apresentaram episódio de TVP. A característica epidemiológica da população estudada foi similar à de outros estudos envolvendo pacientes com câncer em tratamento de TVP (Carrier et al., 2009; Morgan et al., 2002). O tempo médio de tratamento foi de sete meses em ambos os braços. Todas as pacientes fizeram uso de HBPM inicialmente, pois é o tratamento inicial de escolha padrão para TVP na instituição. Pacientes em tratamento de TVP que estavam em quimioterapia também usaram HBPM durante todo o ciclo. Apesar do curto tempo de meia-vida das tecnologias analisadas, o cruzamento das terapias ao longo do tratamento pode ser fator de confundimento na análise dos desfechos selecionados. Dessa forma, considerou-se que o tempo de acompanhamento fosse contabilizado a partir do início do uso da tecnologia com tempo mínimo de três meses de uso.

A construção do modelo de decisão levou em consideração os principais desfechos descritos na literatura. E sua frequência no mundo real demonstrou que as duas tecnologias apresentaram semelhantes resultados de efetividade e segurança e em concordância com os estudos disponíveis em literatura (EINSTEIN Investigators et al., 2010; Carrier et al., 2014; Prandoni et al., 2002; Meyer et al., 2002). Contudo, difere de uma hipótese inicial de que em um estudo de mundo real se esperaria diminuição da efetividade da ENOXA em virtude da apresentação farmacêutica em seringa e, por consequência, menor adesão ao tratamento, o que não ocorreu.

O sangramento é um evento comum em pacientes com câncer. Prandoni et al. (2002) avaliaram a ocorrência de TVP recorrente e sangramento em pacientes com e sem câncer. No subgrupo dos pacientes com câncer ginecológico, foi observada maior taxa de sangramento comparado a outros tipos de câncer (Carrier et al., 2009; Prandoni et al., 2002; Azevedo et al., 2011). Portanto, o uso de anticoagulantes pode afetar a frequência desses desfechos.

Para esta análise, não foi possível avaliar a relação causal entre o câncer e o uso dos anticoagulantes para o desfecho sangramento; dessa forma, foi assumido como pressuposto que todo evento de sangramento estava associado ao uso do anticoagulante. Entretanto, os casos de sangramento maior possuem relevante impacto clínico e alto custo, conforme descrito na literatura (EINSTEIN Investigators et al., 2010; Prandoni et al., 2002; Meyer et al., 2002). Entre os pacientes analisados, nenhum apresentou sangramento maior, sem complicações clínicas relevantes, o que em geral ocasionou

Tabela 4. Análise de sensibilidade univariada e bivariada determinística do modelo

\begin{tabular}{|c|c|c|c|c|c|c|}
\hline & \multicolumn{2}{|c|}{ Cenário 1 (R\$) } & \multicolumn{2}{|c|}{ Cenário 2 (R\$) } & \multicolumn{2}{|c|}{ Cenário 3 (R\$) } \\
\hline & ENOXA & RVXB & ENOXA & RVXB & ENOXA & RVXB \\
\hline Custo & $7.483,25$ & $1.832,09$ & $7.498,05$ & 1470,09 & $7.498,05$ & $2.222,03$ \\
\hline Custo incremental & $5.651,46$ & - & $6.027,96$ & - & $5.276,02$ & - \\
\hline Efetividade & 0,9616 & 0,9743 & 0,9616 & 0,9743 & 0,9616 & 0,9743 \\
\hline Efetividade incremental & $-0,0127$ & - & $-0,0127$ & - & $-0,0127$ & - \\
\hline Custo-efetividade & $7.782,39$ & $1.880,41$ & $7.797,47$ & 1508,86 & $7.797,47$ & $2.280,64$ \\
\hline RCEl & - & DOMINANTE & & DOMINANTE & & DOMINANTE \\
\hline
\end{tabular}

Cenário 1: Aumento em 25\% do custo da RVXB. Cenário 2: Agravamento do desfecho sangramento. Cenário 3: Uso de complexo protrombínico para tratamento de sangramento maior e aumento em 50\% do custo da RVXB. Fonte: Elaboração própria. 
a suspensão temporária do anticoagulante. Todos os eventos analisados foram tratados ambulatorialmente, sem necessidade de internação.

O manejo da TVP recorrente durante o período de anticoagulação não está bem definido. Algumas estratégias incluem aumento de dose, troca do anticoagulante ou inserção de filtro de veia cava. A atual abordagem clínica para TVP recorrente em pacientes com câncer é bastante heterogênea e com pouca descrição do seu manejo (Romualdi \& Ageno, 2016). Para os pacientes avaliados neste estudo, além da investigação por imagem, foi mantido do uso do anticoagulante, com aumento da dose de ENOXA ou troca da RVXB por ENOXA. Somente dois pacientes apresentaram esse evento, porém foi mantido o medicamento em ambos os casos. De acordo com os registros de prontuário, os sinais e sintomas de uma possível TVP recorrente, como dor e edema, também podem indicar progressão da doença.

Considerando os desfechos analisados e os custos associados ao tratamento, o uso da RVXB demonstrou-se mais custo-efetiva para uso ambulatorial. Em virtude da relação de não inferioridade entre as tecnologias e o baixo impacto dos desfechos no modelo proposto, a dominância de uma tecnologia sobre a outra se restringiu ao custo dos medicamentos. Até o momento não foram encontrados estudos que façam esse tipo de análise comparativa de mundo real entre essas tecnologias em pacientes com câncer. Todos os cenários empregados na análise de sensibilidade demonstraram a RVXB com tecnologia dominante.

\section{Conclusão}

Os desfechos de sangramento e TVP recorrente, associados a segurança e a efetividade, apresentaram frequência semeIhante no tratamento com ENOXA ou com RVXB. Considerando o tempo médio de sete meses de tratamento, o custo total de tratamento calculado foi de $R \$ 7.490,49$ e de $\mathrm{R} \$ 1.172,02$ para o tratamento com ENOXA e com RVXB, respectivamente. A ENOXA apresentou custo incremental de $R \$ 6.318,47$ e a análise de sensibilidade demonstrou-se favorável para o uso da RVXB no tratamento de TVP em todos os cenários propostos. Por fim, a RVXB nesse contexto é a tecnologia dominante no tratamento ambulatorial da TVP em pacientes com câncer ginecológico.

\section{Referências bibliográficas}

Azevedo AM, Pereira SCM, Pereira IGA, Schramm M, Arcuri LJ, Tabak DG. Manuseio de complicações hemorrágicas adquiridas no tratamento do paciente oncológico. Onco\&. 2011;5:18-25.

Bates SM, Jaeschke R, Stevens SM, Goodacre S, Wells PS, Stevenson MD, et al. Diagnosis of DVT: Antithrombotic Therapy and Prevention of Thrombosis, 9th ed: American College of Chest Physicians EvidenceBased Clinical Practice Guidelines. Chest. 2012;141(2 Suppl):e351S-e418S.
Bayer. Xarelto. Bula do medicamento. 2015

Brose KMJ, Lee AYY. Cancer-associated thrombosis: prevention and treatment. Curr Oncol. 2008;15(Suppl 1):S58-67.

Carrier M, Cameron C, Delluc A, Castellucci L, Khorana AA, Lee AY. Efficacy and safety of anticoagulant therapy for the treatment of acute cancerassociated thrombosis: a systematic review and meta-analysis. Thromb Res. 2014;134(6):1214-9.

Carrier M, Le Gal G, Cho R, Tierney S, Rodger M, Lee AY. Dose escalation of low molecular weight heparin to manage recurrent venous thromboembolic events despite systemic anticoagulation in cancer patients. J Thromb Haemost. 2009;7(5):760-5.

Diamantopoulos A, Lees M, Wells PS, Forster F, Ananthapavan J, McDonald H. Cost-effectiveness of rivaroxaban versus enoxaparin for the prevention of postsurgical venous thromboembolism in Canada. Thromb Haemost. 2010;104(4):760-70.

Duran A, Ferracini M, Costa A. Análise de custo-efetividade e impacto orçamentário de rivaroxabana no tratamento da trombose venosa profunda sob a perspectiva do sistema de saúde suplementar brasileiro. J Bras Econ Saúde. 2013;5(1):38-44.

EINSTEIN Investigators, Bauersachs R, Berkowitz SD, Brenner B, Buller HR, Decousus $H$, Gallus AS, et al. Oral rivaroxaban for symptomatic venous thromboembolism. N Engl J Med. 2010;363(26):2499-510.

Heit JA, O'Fallon WM, Petterson TM, Lohse CM, Silverstein MD, Mohr DN, et al. Relative impact of risk factors for deep vein thrombosis and pulmonary embolism: a population-based study. Arch Intern Med. 2002;162(11):1245-8.

Kaatz S, Ahmad D, Spyropoulos AC, Schulman S; Subcommittee on Control of Anticoagulation. Definition of clinically relevant non-major bleeding in studies of anticoagulants in atrial fibrillation and venous thromboembolic disease in non-surgical patients: communication from the SSC of the ISTH. J Thromb Haemost. 2015;13(11):2119-26.

Kearon C, Akl EA, Ornelas J, Blaivas A, Jimenez D, Bounameaux H, et al. Antithrombotic therapy for VTE disease: CHEST Guideline and Expert Panel Report. Chest. 2016;149(2):315-52.

Khorana AA, Kuderer NM, Culakova E, Lyman GH, Francis CW. Development and validation of a predictive model for chemotherapy-associated thrombosis. Blood. 2008;111(10):4902-7.

Lee AYY, Peterson EA. Treatment of cancer-associated thrombosis. Blood. 2013;122(14):2310-7.

Lyman GH, Bohlke K, Khorana AA, Kuderer NM, Lee AY, Arcelus II, et al.; American Society of Clinical Oncology. Venous thromboembolism prophylaxis and treatment in patients with cancer: american society of clinical oncology clinical practice guideline update 2014. J Clin Oncol. 2015;33(6):654-6.

Meis E, Levy RA. Câncer e trombose: uma revisão da literatura. Rev Bras Cancerol. 2007;53(2):183-93.

Meyer G, Marjanovic Z, Valcke J, Lorcerie B, Gruel Y, Solal-Celigny P, et al. Comparison of low-molecular-weight heparin and warfarin for the secondary prevention of venous thromboembolism in patients with cancer: a randomized controlled study. Arch Intern Med. 2002;162(15):1729-35.

Ministério da Saúde (Brasil). Secretaria de Ciência, Tecnologia e Insumos Estratégicos. Departamento de Ciência e Tecnologia. Diretrizes metodológicas: Diretriz de Avaliação Econômica/Ministério da Saúde, Secretaria de Ciência, Tecnologia e Insumos Estratégicos, Departamento de Ciência e Tecnologia. 2a ed. Brasília: Ministério da Saúde; 2014.

Morgan MA, lyengar TD, Napiorkowski BE, Rubin SC, Mikuta JJ. The clinical course of deep vein thrombosis in patients with gynecologic cancer. Gynecol Oncol. 2002;84(1):67-71. 
Prandoni P, Lensing AW, Piccioli A, Bernardi E, Simioni P, Girolami B, et al. Recurrent venous thromboembolism and bleeding complications during anticoagulant treatment in patients with cancer and venous thrombosis. Blood. 2002;100(10):3484-8.

Renni MJ, Russomano FB, Mathias LF, Koch HA. Thromboembolic event as a prognostic factor for the survival of patients with stage IIIB cervical cancer. Int J Gynecol Cancer. 2011;21(4):706-10.

Romualdi E, Ageno W. Management of recurrent venous thromboembolism in cancer patients. Thromb Res. 2016;140 Suppl 1:S128-31.

Sanofi-Aventis. Clexane. Bula do medicamento. 2015.

Secoli SR, Nita ME, Ono-Nita SK, Nobre M. Avaliação de Tecnologia em Saúde. II. A análise de custo-efetividade. Arq Gastroenterol. 2010;47(4):329-33.
Silva AP, Santoni NB, Schiola A, Teich V, Pepe C, Laranjeira F. Custo-efetividade e impacto orçamentário da rivaroxabana na prevenção de eventos tromboembólicos em pacientes pós-artroplastia de quadril e joelho em comparação com dabigatrana, enoxaparina e sem profilaxia sob a perspectiva do Sistema de Saúde Suplementar Brasileiro. J Bras Econ Saúde. 2011;3(3):259-68.

Short NJ, Connors JM. New oral anticoagulants and the cancer patient. Oncologist. 2014;19(1):82-93.

Streiff MB, Holmstrom B, Ashrani A, Bockenstedt PL, Chesney C, Eby C, et al. Cancer-Associated Venous Thromboembolic Disease, Version 1.2015. J Natl Compr Canc Netw. 2015;13(9):1079-95. 